\title{
GROUP-GRADED RINGS, SMASH PRODUCTS, AND GROUP ACTIONS
}

BY

\author{
M. COHEN AND S. MONTGOMERY ${ }^{1}$
}

\begin{abstract}
Let $A$ be a $k$-algebra graded by a finite group $G$, with $A_{1}$ the component for the identity element of $G$. We consider such a grading as a "coaction" by $G$, in that $A$ is a $k[G]^{*}$-module algebra. We then study the smash product $A \# k[G]^{*}$; it plays a role similar to that played by the skew group ring $R * G$ in the case of group actions, and enables us to obtain results relating the modules over $A, A_{1}$, and $A \# k[G]^{*}$. After giving algebraic versions of the Duality Theorems for Actions and Coactions (results coming from von Neumann algebras), we apply them to study the prime ideals of $A$ and $A_{1}$. In particular we generalize Lorenz and Passman's theorem on incomparability of primes in crossed products. We also answer a question of Bergman on graded Jacobson radicals.
\end{abstract}

Introduction. The analogy between rings graded by a finite group $G$ and rings on which $G$ acts as automorphisms, in which the identity component in the graded ring corresponds to the fixed ring of the group action, has been noticed by a number of people $[2,6,24]$. In particular, when $G$ is abelian and the ring is an algebra over a field containing a primitive $n$th root of unity, where $n=|G|$, the two notions coincide; for, in that case the ring is graded by $\hat{G}$, the dual group of $G$. Our purpose in this paper is to use the fact that gradings and group actions are dual concepts, even when $G$ is not abelian, in order to obtain new results about graded rings. The idea of duality has already proved very useful in studying von Neumann algebras and $C^{*}$-algebras. The second author wishes to thank G. Pedersen, M. Rieffel and M. Takesaki for informative conversations about duality.

Generally, we first point out that a grading by $G$ can be considered as a "coaction" of $G$. For a $k$-algebra $A$ graded by $G$, we can then form a certain algebra $A \# k[G]^{*}$; this algebra plays the role for graded rings that the skew group algebra $A * G$ plays for group actions, and can be used to form a Morita context relating $A$ and $A_{1}$ (the identity component of $A$ ). We then use the "Duality Theorem for Coactions" to solve problems about graded rings, concerning the Jacobson radical, prime ideals, and semiprimeness, by reducing them to known results about group actions.

Received by the editors September 27, 1982 and, in revised form, January 25, 1983. Some of these results were presented at a Special Session, AMS Meeting in Norman, Oklahoma, March 18, 1983.

1980 Mathematics Subject Classification. Primary 16A03, 16A24, 16A72; Secondary 46L40, 16A66, $16 \mathrm{~A} 12$.

'Both authors received travel support from U.S.-Israel Binational Science Foundation Grant No. 2369/81; the second author also was partially supported by NSF Grant No. 81-01730. 
More specifically, let $A$ be a $k$-algebra with 1 , where $k$ is a commutative ring, and let $G$ be a finite group. In $\S 1$, we show that, just as an action of $G$ as automorphisms on $A$ is equivalent to $A$ being a "module algebra" for the group algebra $k[G]$, a grading of $A$ by $G$ is equivalent to $A$ being a "module algebra" for the dual algebra $k[G]^{*}$; in this sense the grading is a coaction. Thus when $A$ is graded, we may form the smash product $A \# k[G]^{*}$. The formal properties of this algebra are summarized in Proposition 1.4.

In $\S 2$, we consider graded $A$-modules and modules over $A \# k[G]^{*}$ and $A_{1}$. We first show that there is a category isomorphism between graded $A$-modules and $A \# k[G]^{*}$-modules. We then form a Morita context for a $G$-graded algebra $A$, using $A$ as both an $A_{1}-A \# k[G]^{*}$ and an $A \# k[G]^{*}-A_{1}$ bimodule. We show that properties of the Morita context are related to various properties of the grading. In particular, we show that $A$ is strongly $G$-graded (in the sense of Dade [7]) if and only if $A_{1}$ is Morita equivalent to $A \# k[G]^{*}$. We also see that the nondegeneracy condition of Cohen and Rowen [6] corresponds to nondegeneracy of the form (, ) in the Morita context. As a consequence, we show that $A \# k[G]^{*}$ is semiprime if and only if $A$ is graded semiprime. This is analogous to the theorem of Fisher and Montgomery [11] for skew group rings.

In $\S 3$, we give proofs of the two duality theorems. These theorems are essentially translations of known results in von Neumann algebras [14, 19, 27], but our proofs are elementary. The Duality Theorem for Actions says that if $G$ acts on $A$, then $A * G$ is graded by $G$ and $(A * G) \# k[G]^{*} \cong M_{n}(A)$, the $n \times n$ matrix ring over $A$, where $n=|G|$. The Duality Theorem for Coactions says that if $A$ is graded by $G$, then there is an action of $G$ on $A \# k[G]^{*}$, and $\left(A \# k[G]^{*}\right) * G \cong M_{n}(A)$.

In $\S \S 4-7, A$ is graded by $G$. In $\S 4$, we consider Jacobson radicals, and show that the graded Jacobson radical $J_{G}(A)$ is always contained in the usual Jacobson radical $J(A)$. This answers a question of $\mathrm{G}$. Bergman [3]. We also show that $J\left(A \# k[G]^{*}\right)=$ $J_{G}(A) \# k[G]^{*}$, and that $J\left(A_{1}\right)=J(A) \cap A_{1}$.

In $\$ 5$ we show similar results for the prime radical $N(A)$. We also show that when $A$ has no $|G|$-torsion, $A$ is semiprime if and only if it is graded semiprime.

In $\$ 6$ we compare the prime ideals of $A$ and $A \# k[G]^{*}$, obtaining results similar to those of Lorenz and Passman for crossed products [15]. In particular we show (Theorem 6.3) that if $Q$ is a graded prime of $A$, then $A$ has $m \leqslant|G|$ primes minimal over $Q$, say $P_{1}, \ldots, P_{m}$; these are precisely the primes satisfying $P \cap A_{1}=Q \cap A_{1}$, and if $I=P_{1} \cap \cdots \cap P_{m}$, then $I^{\mid G} \subseteq Q$.

In $\S 7$, the last section, we first prove incomparability for primes of $A$ and $A_{1}$ : if $P \subsetneq Q$ are primes of $A$, then $P \cap A_{1} \subsetneq Q \cap A_{1}$. This generalizes Lorenz and Passman's theorem on incomparability of primes in crossed products [15], since a crossed product is a group-graded ring.

We then compare the primes of $A$ and $A_{1}$ by a method similar to that used in [18]. We show that if $P$ is a prime in $A$, then there exist $k \leqslant|G|$ primes of $A_{1}$ minimal over $P \cap A_{1}$; conversely, given a prime $p$ of $A_{1}, p$ determines a unique graded prime $Q$ of $A$ so that $p$ is minimal over $Q \cap A_{1}$ (Theorem 7.2). Finally, we apply these results to show that an "additivity principle" for Goldie ranks holds between primes of $A$ and $A_{1}$. 
Some of the results in $\$ \S 4-7$ were known previously for the special case of strongly graded rings [21].

Before beginning, we fix our notation. $A$ will always denote a $k$-algebra with 1 , over a commutative ring $k$ with 1 , and $G$ is a finite group. $A$ is graded by $G$ if $A=\Sigma_{g \in G} \oplus A_{g}$, where the $A_{g}$ are $k$-subspaces of $A$ and $A_{g} A_{h} \subseteq A_{g h}$, for all $g, h \in G$. For any ideal $I$ of $A$ (left, right, or two-sided), we define

$$
I_{G}=\sum_{g \in G} \oplus\left(I \cap A_{g}\right) .
$$

We say that $I$ is graded if $I=I_{G}$; more generally, $I_{G}$ is the largest graded ideal of $A$ contained in $I$.

Other definitions will be made as we need them.

1. Smash products, group actions, and coactions. In this section we discuss the duality of group actions and gradings, using some ideas from the theory of Hopf algebras. The advantage of this approach is that it demonstrates that the algebra $A \# k[G]^{*}$ mentioned in the introduction arises naturally from a group grading, and in fact it is the analog of the skew group ring $R * G$ constructed for a group $G$ acting on a ring $R$ as automorphisms.

A standard reference on Hopf algebras is Sweedler's book [28], and we shall follow his notation.

Let $A$ be a $k$-algebra and $H$ a $k$-bialgebra; that is $H$ is an algebra in the usual sense, but in addition has a comultiplication $\Delta: H \rightarrow H \otimes_{k} H$ and a counit $\varepsilon: H \rightarrow k$, satisfying appropriate properties [28, p. 53]. For any $h \in H$, we use the standard notation $\Delta(h)=\Sigma_{(h)} h_{(1)} \otimes h_{(2)}$.

In order to form the smash product $A \# H$, the required condition is that $A$ is an $H$-module algebra [28, pp. 138, 153]:

Definition 1.1. Let $A$ be a $k$-algebra and $H$ a $k$-bialgebra, with comultiplication $\Delta$ and counit $\varepsilon$. Then $A$ is an $H$-module algebra if there exists a map $\psi: H \otimes A \rightarrow A$ satisfying

(1) $A$ is an $H$-module under $\psi$,

(2) $\psi(h \otimes a b)=\Sigma_{(h)} \psi\left(h_{(1)} \otimes a\right) \psi\left(h_{(2)} \otimes b\right)$, for $a, b \in A, h \in H$, and $\Delta(h)$ as above,

(3) $\psi(h \otimes 1)=\varepsilon(h) 1_{A}$.

For simplicity, we will write $h \cdot a$ for $\psi(h \otimes a)$. Thus for example, condition (2) above becomes $h \cdot a b=\Sigma_{(h)}\left(h_{(1)} \cdot a\right)\left(h_{(2)} \cdot b\right)$.

Now let $A$ be an $H$-module algebra. One can then define the smash product $A \# H$, as follows: as a vector space, $A \# H$ is $A \otimes_{k} H$, with elements $a \otimes h$ written as $a \# h$. Multiplication is defined by

$$
(a \# g)(b \# h)=\sum_{(g)} a\left(g_{(1)} \cdot b\right) \#\left(g_{(2)} h\right), \quad \text { where } \Delta(g)=\sum_{(g)} g_{(1)} \otimes g_{(2)} .
$$

This makes $A \# H$ into a $k$-algebra with unit element $1=1_{A} \# 1_{H}[28$, p. 156].

We first consider the relationship between group actions and $k[G]$-module algebras. Note that $k[G]$ is a bialgebra, with comultiplication given by $\Delta(g)=g \otimes g$ and counit given by $\varepsilon(g)=1$, for any $g \in G$. By an action of $G$ on a $k$-algebra $A$, we 
mean a group homomorphism $\alpha: G \rightarrow \operatorname{Aut}_{k}(A)$; let $\alpha_{g}$ denote the image of $g$ in Aut $_{k}(G)$. The following proposition is presumably well known; certainly the first part appears in [28].

Proposition 1.2. Any action of $G$ on $A$ makes $A$ into a $k[G]$-module algebra. Conversely, if $A$ is a $k[G]$-module algebra, this arises from an action of $G$ on $A$.

Proof. If $\alpha: G \rightarrow \operatorname{Aut}_{k}(A)$ is an action of $G$ on $A$, then (as in [28, p. 154]) the map $g \otimes a \rightarrow \alpha_{g}(a)$ makes $A$ into a $k[G]$-algebra. Conversely, say that $A$ is a $k[G]$-module algebra and write $\psi(g \otimes a)=g \cdot a$. Since $A$ is a $k[G]$-module, if $a, b \in A$, $g \in G$, then $a=1 \cdot a=g^{-1} \cdot(g \cdot a)=g \cdot\left(g^{-1} \cdot a\right)$, and $g \cdot(a+b)=g \cdot a+g \cdot$ $b$. Thus if we set $\alpha_{g}(a)=g \cdot a, \alpha_{g} \in \operatorname{End}_{k}(A)$ is a bijection. Using $\Delta(g)=g \otimes g$ and part (2) of the definition, $g \cdot a b=(g \cdot a)(g \cdot b)$, and thus $\alpha_{g}$ is an automorphism of $A$. Finally, the map $\alpha: G \rightarrow \operatorname{Aut}_{k}(A)$ given by $g \rightarrow \alpha_{g}$ is a group homomorphism since $\alpha_{g}\left(\alpha_{h}(a)\right)=g \cdot(h \cdot a)=(g h) \cdot a=\alpha_{g h}(a)$, any $\alpha \in A$. Thus $\alpha$ is an action of $G$ on $A$.

For the case of group actions, we see that the smash product $A \# k[G]$ has multiplication $(a \# g)(b \# h)=a \alpha_{g}(b) \# g h$. Thus, it is just the familiar skew group ring, or trivial crossed product, and we will denote it by $A * G$.

We now turn to the dual algebra $k[G]^{*}$, and show its close connection to $G$-graded algebras. A $k$-basis of $k[G]^{*}$ is the set of "projections" $\left\{p_{g} \mid g \in G\right\}$; that is, for any $g \in G$ and $x=\Sigma_{h \in G} \alpha_{h} h \in k[G], p_{g}(x)=\alpha_{g} \in k$. The set $\left\{p_{g}\right\}$ consists of orthogonal idempotents whose sum is 1 . The comultiplication on $k[G]^{*}$ is given by $\Delta\left(p_{g}\right)=\Sigma_{h \in G} p_{g h^{-1}} \otimes p_{h}$, and the counit is given by $\varepsilon\left(p_{g}\right)=\delta_{1, g}$ (where $\delta$ denotes the Kronecker delta).

We observe that $A$ being graded by $G$ is equivalent to the existence of a map $\beta: G \rightarrow \operatorname{End}_{k}(A)$ satisfying the following properties (where $\beta_{g}$ denotes the image of $g$ in $\left.\operatorname{End}_{k}(A)\right)$ :

(1) for all $g, h \in G, \beta_{g} \circ \beta_{h}=0$ if $g \neq h$, and $\beta_{g} \circ \beta_{g}=\beta_{g}$,

(2) $\Sigma_{g \in G} \beta_{g}=I$, the identity mapping,

(3) for each $g \in G, a, b \in A, \beta_{g}(a b)=\Sigma_{h \in G} \beta_{g h^{-1}}(a) \beta_{h}(b)$.

The first part of the next proposition was observed by Bergman [2].

Proposition 1.3. If $A$ is graded by $G$, then $A$ is a $k[G]^{*}$-module algebra. Conversely, if $A$ is a $k[G]^{*}$-module algebra, then $A$ is graded by $G$.

Proof. Assume that $A$ is graded, so $A=\Sigma_{g \in G} \oplus A_{g}$. Any $a \in A$ may be written uniquely as $a=\Sigma_{g} a_{g}$, where $a_{g} \in A_{g}$. We define the action of $k[G]^{*}$ on $A$ by $p_{g} \cdot a=a_{g}$, where the $\left\{p_{g}\right\}$ are the dual basis for $k[G]^{*}$. That is, $p_{g}$ is the projection onto the " $g$ th" part of any element of $A$. Using the fact that $p_{h} p_{g}=\delta_{h, g} p_{g}$, it is clear that $A$ is a $k[G]^{*}$-module. Also, this action satisfies (2) of Definition 1.1. For, say $x, y \in A$ and $p_{g} \in k[G]^{*}$; then

$$
p_{g} \cdot(x y)=(x y)_{g}=\sum_{h \in G} x_{g h^{-1}} y_{h}=\sum_{h \in G}\left(p_{g h^{-1}} \cdot x\right)\left(p_{h} \cdot y\right)
$$

which is compatible with $\Delta\left(p_{g}\right)=\sum_{h \in G} p_{g h^{-1}} \otimes p_{h}$. Property (3) is trivial. Thus $A$ is a $k[G]^{*}$-module algebra. 
Conversely, say that $A$ is a $k[G]^{*}$-module algebra, and denote the action of $k[G]^{*}$ on $A$ by $p_{g} \cdot a$, for any $a$. Let $A_{g}=\left\{p_{g} \cdot a\right.$, all $\left.a \in A\right\}$; since $p_{g} p_{h}=\delta_{g, h} p_{g}$ and $\Sigma_{g} p_{g}=1$, it is clear that $A=\Sigma_{g \in G} \oplus A_{g}$. Since the given action satisfies (2) of Definition 1.1, and $\Delta\left(p_{g}\right)=\sum_{h \in G} p_{g h^{-1}} \otimes p_{h}$, it follows that $p_{g} \cdot(x y)=$ $\Sigma_{g \in G}\left(p_{g h^{-1}} \cdot x\right)\left(p_{h} \cdot y\right)$. Thus $A_{g h^{-1}} A_{h} \subseteq A_{g}$, for any $g, h \in G$, so that $A$ is graded.

When $A$ is graded, we may thus construct the smash product $A \# k[G]^{*}$. For $a, b \in A$, and basis elements $p_{g}, p_{h} \in k[G]^{*}$, the product is given by

$$
\left(a \# p_{g}\right)\left(b \# p_{h}\right)=\sum_{l \in G} a\left(p_{g l^{-1}} \cdot b\right) \#\left(p_{l} p_{h}\right)=a\left(b_{g h^{-1}}\right) \# p_{h}
$$

using $\Delta\left(p_{g}\right)=\Sigma_{l \in G} p_{g l^{-1}} \otimes p_{l}$, the fact that the $\left\{p_{l}\right\}$ are orthogonal idempotents, and the fact that $p_{g h^{-1}} \cdot b=b_{g h^{-1}}$ by the module action in Proposition 1.3.

This notation may be simplified slightly. For, $(a \# 1)\left(1 \# p_{h}\right)=\left(a \# \sum_{g} p_{g}\right)\left(1 \# p_{h}\right)$ $=a\left(\sum_{g} 1_{g h^{-1}}\right) \# p_{h}=a \# p_{h}$. That is, $A$ may be identified with $A \# 1$, and $k[G]^{*}$ with $1 \# k[G]^{*}$ in $A \# k[G]^{*}$. We may therefore write the above multiplication more simply as:

$$
\left(a p_{g}\right)\left(b p_{h}\right)=a b_{g h^{-1}} p_{h} .
$$

We summarize our description of $A \# k[G]^{*}$.

Proposition 1.4. Let $A$ be graded by the finite group $G$. Then $A \# k[G]^{*}$ is the free right and left $A$-module with basis $\left\{p_{g} \mid g \in G\right\}$, a set of orthogonal idempotents whose sum is 1 , and with multiplication given by $(*)$ above. In particular,

(1) for $a \in A, p_{h} a=\Sigma_{g} a_{h g^{-1}} p_{g}$,

(2) for $a_{g} \in A_{g}, p_{h} a_{g}=a_{g} p_{g^{-1} h}$,

(3) each $p_{h}$ centralizes $A_{1}$.

Proof. The $\left\{p_{g}\right\}$ are a free $k$-basis for $k[G]^{*}$, and so $\left\{1 \# p_{g}\right\}$ are a free left $A$-basis for $A \# k[G]^{*}$. Using (1), it is clear they are also a free right $A$-basis. Since the $\left\{p_{g}\right\}$ are orthogonal idempotents in $k[G]^{*}$, and $\left(1 \# p_{g}\right)\left(1 \# p_{n}\right)=1_{g h^{-1}} \# p_{g}=$ $\delta_{g, h} \# p_{g}$, the $\left\{1 \# p_{g}\right\}$ are also orthogonal idempotents in $A \# k[G]^{*}$.

(1) Using (*), $p_{h} a=\left(1 \# p_{h}\right)\left(a \# \Sigma_{g} p_{g}\right)=\Sigma_{g \in G} a_{h g^{-1}} \# p_{g}$.

(2) By (1), $p_{h} a_{g}=\sum_{t \in G}\left(a_{g}\right)_{h t^{-1}} \# p_{t}$. However, $\left(a_{g}\right)_{h t^{-1}}=0$ unless $h t^{-1}=g$, in which case $t=g^{-1} h$ and $\left(a_{g}\right)_{g}=a_{g}$. Hence $p_{h} a_{g}=a_{g} p_{g^{-1} h}$.

(3) Clearly if $g=1, p_{h} a_{1}=a_{1} p_{h}$.

Corollary 1.5. Let $A$ be G-graded, and $A \# k[G]^{*},\left\{p_{g}\right\}$ be as above. Let I be any graded ideal of $A$. Then

(1) $p_{h}\left(I \# k[G]^{*}\right) p_{g}=I_{h g^{-1}} p_{g}=p_{h} I p_{g}$,

(2) $p_{1}\left(I \# k[G]^{*}\right) p_{1}=I_{1} p_{1}$, which is isomorphic as a ring to $I_{1}$.

Proof. (1) Choose $a \in I, p_{s} \in k[G]^{*}$. Then $p_{h}\left(a p_{s}\right) p_{g}=0$ unless $s=g$. In that case, using $(*), p_{h}\left(a p_{g}\right) p_{g}=p_{h} a p_{g}=a_{h g^{-1}} p_{g}$. Thus $p_{h}\left(I \# k[G]^{*}\right) p_{g} \subseteq I_{h g^{-1}} p_{g}$; by reversing the argument, equality follows.

(2) The first statement follows using $g=h=1$. Using the fact that $p_{1}$ centralizes $I_{1}$ (Proposition 1.4(3)), $\left(a p_{1}\right)\left(b p_{1}\right)=a b p_{1}$, for $a, b \in I_{1}$, and thus $I_{1} \cong I_{1} p_{1}$. 
REMARK 1.6. We note here that several of the ideas above have appeared in work on operator algebras, in somewhat different form. When $A$ is a von Neumann algebra, $G$ a locally compact group, and $\mathscr{R}(G)$ is the von Neumann algebra on $L^{2}(G)$ generated by the regular representation of $G$, a coaction of $G$ on $A$ is defined to be a ring isomorphism $\beta$ of $A$ into the closure of $A \otimes \Re(G)$ satisfying $\left(\beta \otimes 1_{A}\right) \circ \beta=\left(1_{A} \otimes \Delta\right) \circ \beta[\mathbf{2 0}]$. When $G$ is finite, one can show that this definition is equivalent to the existence of a grading of $A$ by $G$. To be consistent with the terminology of [20], and also in light of Propositions 1.2 and 1.3, we will sometimes refer to a grading of $A$ by $G$ as a coaction of $G$ on $A$.

Now say that there is a coaction of the locally compact group $G$ on the von Neumann algebra $A$. Then, as in [20], one may construct the "crossed product with respect to a coaction", $A \times{ }_{\beta} G$. When $G$ is a finite group, it is possible to show that $A \times{ }_{\beta} G$ is simply our algebra $A \# k[G]^{*}$ as given in Proposition 1.4. In a somewhat different direction, we consider a Banach *-algebraic bundle $B$ over a locally compact group $G$, as defined by J. M. G. Fell [9]; among other things, this means that $B$ is a $\mathbb{C}$-algebra graded by $G$. Given a locally compact Hausdorff space $M$ on which $G$ acts continuously, he constructs a new Banach *-algebraic bundle $D$ over $G$, called the $G, M$ transformation bundle derived from $B$ [9, p. 260]. Once again, when $G$ is finite and $M=\mathbb{C}[G]$ (considered as a vector space, on which $G$ acts by left multiplication), $D$ can be shown to be our construction $B \# \mathbb{C}[G]^{*}$.

2. Modules, Morita contexts, and gradings. In this section we are concerned with the relationships between modules for $A, A_{1}$, and $A \# k[G]^{*}$. We first establish a category isomorphism between $A \# k[G]^{*}$-modules and graded $A$-modules, and a Maschke-type theorem for $A \# k[G]^{*}$. We then turn to Morita contexts. For a finite group $G$ acting on a commutative ring $A$, Chase, Harrison, and Rosenberg [4] showed that there is a Morita context $\left[A^{G}, V, W, A * G\right]$ associated with the fixed ring $A^{G}$ and the skew group ring $A * G$, using $V={ }_{A^{G}} A_{A * G}$ and $W={ }_{A * G} A_{A^{G}}$. This context was studied for noncommutative rings by M. Cohen in [5]. We show in this section that an analogous situation holds for coactions: if $A$ is graded by $G$, there is a Morita context associated to $A \# k[G]^{*}$ and $A_{1}$. We then show that several properties of the gradings can be interpreted as properties of the Morita context. For a general reference on Morita contexts, see [1].

For any ring $R$, let $\operatorname{Mod}(R)$ denote the category of all unital right $R$-modules and their $R$-homomorphisms. If the ring $A$ is graded by $G$, a right $A$-module $V$ is graded if $V=\Sigma_{g \in G} \oplus V_{g}$ and if $V_{g} A_{h} \subseteq V_{g h}$, for all $g, h \in G$. One can then form a category $\operatorname{Gr} \operatorname{Mod}(A)$, whose objects are the graded $A$-modules and whose morphisms $f: V \rightarrow W$ are morphisms in $\operatorname{Mod}(A)$ such that $f\left(V_{g}\right) \subseteq W_{g}$ [7, p. 244].

We now consider the relationship between $\operatorname{Gr} \operatorname{Mod}(A)$ and $\operatorname{Mod}\left(A \# k[G]^{*}\right)$.

Lemma 2.1. (1) Let $V \in \operatorname{Mod}\left(A \# k[G]^{*}\right)$. Then $V$ becomes a graded $A$-module by defining $V_{g}=V \cdot p_{g^{-1}}$.

(2) Let $V \in \operatorname{Gr} \operatorname{Mod}(A)$. Then $V$ becomes an $A \# k[G]^{*}$-module by defining, for $v \in V, a \in A, p_{h} \in k[G]^{*}$

$$
v \cdot\left(a p_{h}\right)=(v \cdot a)_{h^{-1}} .
$$


Proof. (1) Clearly $V=\Sigma_{g \in G} \oplus V_{g}$, since the $\left\{p_{g}\right\}$ are orthogonal idempotents whose sum is 1 . Now choose $v_{g} \in V_{g}$, so $v_{g}=v p_{g^{-1}}$, some $v \in V$. If $a_{h} \in A_{h}$, then

$$
v_{g} \cdot a_{h}=v \cdot\left(p_{g^{-1}} a_{h}\right)=v \cdot a_{h} p_{h^{-1} g^{-1}}=\left(v a_{h}\right) \cdot p_{(g h)^{-1}} \in V_{g h} .
$$

Thus $V$ is a graded $A$-module.

(2) Choose $a, b \in A, v \in V, p_{h}, p_{g} \in k[G]^{*}$. Then

$$
\begin{aligned}
v \cdot\left[\left(a p_{h}\right)\left(b p_{g}\right)\right] & =v \cdot\left(a b_{h g^{-1}} p_{g}\right)=\left(v a b_{h g^{-1}}\right)_{g^{-1}}=(v a)_{h^{-1}} b_{h g^{-1}} \\
& =\left((v a)_{h^{-1}} b\right)_{g^{-1}}=(v a)_{h^{-1}} \cdot b p_{g}=\left(v \cdot a p_{h}\right) b p_{g} .
\end{aligned}
$$

Finally, $v \cdot 1=v \cdot \Sigma_{g} p_{g}=\Sigma_{g} v_{g^{-1}}=v$.

Thus $V$ becomes an $A \# k[G]^{*}$-module.

Given $V \in \operatorname{Gr} \operatorname{Mod}(A)$, we define $V^{\#}$ to be $V$ considered as an $A \# k[G]^{*}$-module as in the lemma. For any morphism $f: V \rightarrow W$ in $\operatorname{Gr} \operatorname{Mod}(A)$, define $f^{\#}: V^{\#} \rightarrow W^{\#}$ by setting $f^{\#}=f$. Similarly, given $V \in \operatorname{Mod}\left(A \# k[G]^{*}\right)$, we define $V_{\mathrm{Gr}}$ to be $V$ considered as a graded $A$-module, and for a morphism $f: V \rightarrow W$ in $\operatorname{Mod}\left(A \# k[G]^{*}\right)$, define $f_{\mathrm{Gr}}: V_{\mathrm{Gr}} \rightarrow W_{\mathrm{Gr}}$ by setting $f_{\mathrm{Gr}}=f$.

THEOREM 2.2. Let $A$ be graded by $G$. Then there is a category isomorphism between $\operatorname{Gr} \operatorname{Mod}(A)$ and $\operatorname{Mod}\left(A \# k[G]^{*}\right)$ given by the functors

$$
\begin{aligned}
& ()_{\mathrm{Gr}}: \operatorname{Mod}\left(A \# k[G]^{*}\right) \rightarrow \mathrm{Gr} \operatorname{Mod}(A), \\
& ()^{\#}: \operatorname{Gr} \operatorname{Mod}(A) \rightarrow \operatorname{Mod}\left(A \# k[G]^{*}\right) .
\end{aligned}
$$

Proof. We use the definition of category isomorphism as given in [8, p. 65]. We first show that the maps ( $)_{\mathrm{Gr}}$ and ( $)^{\#}$ are indeed functors. To see this it suffices to show that $f_{\mathrm{Gr}}: V_{\mathrm{Gr}} \rightarrow W_{\mathrm{Gr}}$ is a morphism in $\operatorname{Gr} \operatorname{Mod}(A)$ and that $f^{\#}: V^{\#} \rightarrow W^{\#}$ is a morphism in $\operatorname{Mod}\left(A \# k[G]^{*}\right)$.

We first consider $f_{\mathrm{Gr}}$, where we are given a morphism $f: V \rightarrow W$ in $\operatorname{Mod}\left(A \# k[G]^{*}\right)$. Since $V_{\mathrm{Gr}}$ is graded, choose $v_{g} \in V_{g}$; by construction $v_{g} p_{g^{-1}}=v_{g}$. Thus $f\left(v_{g}\right)=$ $f\left(v_{g} p_{g^{-1}}\right)=f\left(v_{g}\right) p_{g^{-1}} \in W_{g}$. Thus $f_{\mathrm{Gr}}$ is a graded morphism.

Next consider $f^{\#}$, where we are given a morphism $f: V \rightarrow W$ in $\operatorname{Gr} \operatorname{Mod}(A)$. For $v \in V_{g}$ and $a p_{h} \in A \# k[G]^{*}$,

$$
f\left(v_{g} \cdot a p_{h}\right)=f\left(\left(v_{g} \cdot a\right)_{h^{-1}}\right)=\left(f\left(v_{g} \cdot a\right)\right)_{h^{-1}}=\left(f\left(v_{g}\right) \cdot a\right)_{h^{-1}}=f\left(v_{g}\right) \cdot a p_{h} .
$$

Thus $f^{\#}$ is an $A \# k[G]^{*}$-morphism.

To see that these functors give a category isomorphism, we show $\left(()^{\#}\right)_{\mathrm{Gr}}=1$ and $\left(()_{\mathrm{Gr}}\right)^{\#}=1$. For the first, consider $V \in \operatorname{Mod}\left(A \# k[G]^{*}\right)$. In $V_{\mathrm{Gr}}$, choose $v_{g}=v_{g} p_{g^{-1}}$, and let $a p_{h} \in A \# k[G]^{*}$. Then in $\left(V_{\mathrm{Gr}}\right)^{\# \text {, }}$

$$
\left(v_{g} \cdot a p_{h}\right)=\left(v_{g} \cdot a\right)_{h^{-1}}=\left(\left(v_{g}\right) \cdot a\right) \cdot p_{h}=v_{g}\left(a p_{h}\right),
$$

the usual action in $\operatorname{Mod}\left(A \# k[G]^{*}\right)$. Thus $\left(()_{\mathrm{Gr}}\right)^{\#}=1$.

A similar argument shows that $\left(()^{\#}\right)_{\mathrm{Gr}}=1$.

We note that the theorem is also true for left modules: if $V$ is a left $A \# k[G]^{*}$ module, it becomes a left graded $A$-module by defining $V_{g}=p_{g} V$; if $V$ is a left graded $A$-module, it becomes a left $A \# k[G]^{*}$-module by defining $a p_{h} \cdot v=a v_{h}$. The analogs of Lemma 2.1 and Theorem 2.2 follow. 
We next prove a Maschke-type theorem, which unlike the corresponding result for group actions, does not require any assumptions about the characteristic of $A$.

THEOREM 2.3. Let $V$ be a right (left) $A \# k[G]^{*}$-module, and let $W$ be an $A \# k[G]^{*}$ submodule of $V$ which is an $A$-direct summand of $V$. Then $W$ is an $A \# k[G]^{*}$-direct summand of $V$.

Proof. We first consider right modules. Let $\pi: V \rightarrow W$ denote the natural $A$-module projection of $V$ onto $W$. Define $\lambda: V \rightarrow W$ by $\lambda(v)=\sum_{h \in G}\left(v p_{h}\right)^{\pi} p_{h}$.

Now $\left.\lambda\right|_{W}=1$, for if $w \in W$, then $w p_{h} \in W$, all $h \in G$; hence $\left(w p_{h}\right)^{\pi}=w p_{h}$, so $\lambda(w)=\Sigma_{h \in G} w p_{h}^{2}=w\left(\sum_{h} p_{h}\right)=w$. Also, $\lambda$ is an $A \# k[G]^{*}$-module homomorphism. For if $v \in V, a p_{g} \in A \# k[G]^{*}$, using Proposition 1.4

$$
\begin{aligned}
\lambda(v) a p_{g} & =\left[\sum_{h \in G}\left(v \cdot p_{h}\right)^{\pi} p_{h}\right] a p_{g}=\sum_{h \in G}\left(v \cdot p_{h}\right)^{\pi}\left(a_{h g^{-1}} p_{g}\right) \\
& =\sum_{h \in G}\left(v \cdot p_{h} a_{h g^{-1}}\right)^{\pi} p_{g}=\sum_{h \in G}\left(v \cdot a_{h g^{-1}} p_{g}\right)^{\pi} p_{g} \\
& =\left[v \cdot\left(\sum_{h} a_{h g^{-1}}\right) p_{g}\right]^{\pi} p_{g}=\left(v \cdot a p_{g}\right)^{\pi} p_{g} \\
& =\sum_{h \in G}\left(v \cdot a p_{g} p_{h}\right)^{\pi} p_{h}=\lambda\left(v \cdot a p_{g}\right) .
\end{aligned}
$$

Thus $\lambda$ is an $A \# k[G]^{*}$ projection of $V$ onto $W$, hence $W$ is an $A \# k[G]^{*}$-direct summand of $V$.

A very similar argument works for left modules; just define $\lambda: V \rightarrow W$ by $\lambda(v)=\sum_{h \in G} p_{h}\left(p_{h} \cdot v\right)^{\pi}$.

By Lemma 2.1, Theorem 2.3 is equivalent to the following (which could also be proved directly):

THEOREM 2.3'. Let $V$ be a graded (right) A-module, and $W$ a graded submodule of $V$ which has a complement as an A-submodule of $V$. Then $W$ has a graded complement.

We now proceed to the Morita context.

By Lemma $2.1, A$ is a right $A \# k[G]^{*}$-module via $a \cdot b p_{h}=(a b)_{h^{-1}}$, and by the remark following Theorem $2.2, A$ is a left $A \# k[G]^{*}$-module via $b p_{h} \cdot a=b a_{h}$. It is also certainly a right and left $A_{1}$-module via right and left multiplication. We may thus consider the two bimodules

$$
V={ }_{A_{1}} A_{A \# k[G]^{*}} \text { and } W={ }_{A \# k[G]^{*}} A_{A_{1}} .
$$

Let [ , ]: $W \otimes_{A_{1}} V \rightarrow A \# k[G]^{*}$ be defined by $[w, v]=w p_{1} v$.

Let $():, V \otimes_{A \# k[G]^{*}} W \rightarrow A_{1}$ be defined by $(v, w)=(v w)_{1}$.

Proposition 2.4. Let $A$ be graded by $G$. Then $\left[A_{1}, V, W, A \# k[G]^{*}\right]$ forms a Morita context, where $V, W,[$,$] , and (, ) are as defined above.$

Proof. To satisfy the conditions for a Morita context [1], we must show that [, ] is an $A \# k[G]^{*}$-bimodule map which is middle $A_{1}$-linear, that $($,$) is an A_{1^{-}}$ bimodule map which is middle $A \# k[G]^{*}$-linear and that the "associativity" 
conditions hold. We first show the associativity conditions. Say that $v, v^{\prime} \in V, w$, $w^{\prime} \in W$; we need $v \cdot\left[w, v^{\prime}\right]=(v, w) \cdot v^{\prime}$ and $[w, v] \cdot w^{\prime}=w \cdot(v, w)$. Now $v$. $\left[w, v^{\prime}\right]=v \cdot w p_{1} v^{\prime}=\left(v \cdot w p_{1}\right) \cdot v^{\prime}=(v w)_{1} \cdot v^{\prime}=(v, w) \cdot v^{\prime}$. Also $[w, v] \cdot w^{\prime}=$ $w p_{1} v \cdot w^{\prime}=w \cdot\left(p_{1} \cdot\left(v w^{\prime}\right)\right)=w \cdot\left(v w^{\prime}\right)_{1}=w \cdot\left(v, w^{\prime}\right)$.

To check the bimodule maps, by additivity it will suffice to check them on generators. We first consider [ , ]. It is clearly middle $A_{1}$-linear, since $p_{1}$ commutes with elements of $A_{1}$ by Proposition 1.4. Now say that $a p_{h} \in A \# k[G]^{*}, v \in V$, $w \in W$. Then

$$
a p_{h}[w, v]=a p_{h} w p_{1} v=a w_{h} p_{1} v=\left[a w_{h}, v\right]=\left[a p_{h} \cdot w, v\right]
$$

and

$[w, v] \cdot a p_{h}=w p_{1} v a p_{h}=w(v a)_{h^{-1}} p_{h}=w p_{1}(v a)_{h^{-1}}=\left[w,(v a)_{h^{-1}}\right]=\left[w, v \cdot a p_{h}\right]$.

Now for (, ). It is clearly an $A_{1}$-bimodule map, so it suffices to show it is middle $A \# k[G]^{*}$-linear. Again, say $a p_{h} \in A \# k[G]^{*}, v \in V, w \in W$. Then

$$
\left(v \cdot a p_{h}, w\right)=\left((v a)_{h^{-1}} w\right)_{1}=(v a)_{h^{-1}} w_{h}=\left(v a w_{h}\right)_{1}=\left(v, a w_{h}\right)=\left(v, a p_{h} \cdot w\right) .
$$

The proposition is proved.

We now consider nondegeneracy of the two forms [, ] and (, ). Since $A$ has a 1, it is clear that $[]:, V \otimes W \rightarrow A \# k[G]^{*}$ is always nondegenerate: for, if $[V, w]=0$, then $1 p_{1} w=0$, so $w=0$, and similarly if $[v, W]=0$. However, the other form can be degenerate. In [6], Cohen and Rowen obtain a number of consequences of the nondegeneracy of the form (, ).

We now define three successively stronger properties that a grading may have, related to the Morita context. The first is motivated by the work of Cohen and Rowen mentioned above, and the third is due to Dade [7] and Fell [9].

DeFinition. Let $A$ be graded by the group $G$.

(1) The grading is nondegenerate if $($,$) is nondegenerate.$

(2) The grading is faithful if $A$ is a faithful left and right $A \# k[G]^{*}$-module.

(3) $A$ is strongly $G$-graded if $A_{g} A_{g^{-1}}=A_{1}$, for all $g \in G$.

Fell calls a graded ring saturated if it satisfies (3).

We give a more concrete interpretation of (1) and (2).

LEMmA 2.5. (1) The grading on $A$ is nondegenerate $\Leftrightarrow$ for any $0 \neq a_{g} \in A_{g}$, $a_{g} A_{g^{-1}} \neq 0$ and $A_{g^{-1}} a_{g} \neq 0$.

(2) The grading on $A$ is faithful $\Leftrightarrow$ for any $0 \neq a_{g} \in A_{g}, a_{g} A_{h} \neq 0$ and $A_{h} a_{g} \neq 0$, for $g, h \in G$.

Proof. (1) Say that the grading is nondegenerate, but $a_{g} A_{g^{-1}}=0$, some $a_{g} \in A_{g}$. Then $\left(a_{g} A\right)_{1}=\left(a_{g} \Sigma_{h} A_{h}\right)_{1}=a_{g} A_{g^{-1}}=0$, and so $\left(a_{g}, A\right)=0$. By nondegeneracy, $a_{g}=0$. Similarly $A_{g^{-1}} a_{g} \neq 0$.

Conversely, assume the condition, and say that $0=(a, A)=(a A)_{1}$, some $a \in A$. Write $a=\Sigma_{g} a_{g}$, where $a_{g} \in A_{g}$. If $a \neq 0$, then $a_{h} \neq 0$ for some $h \in G$. Since $(a A)_{1}=0$, certainly $\left(a A_{h^{-1}}\right)_{1}=0$. But $\left(a A_{h^{-1}}\right)_{1}=a_{h} A_{h^{-1}}=0$, a contradiction.

(2) Say that $A$ is a faithful right $A \# k[G]^{*}$-module (a similar argument will work on the left) but $A_{h} a_{g}=0$, some $0 \neq a_{g} \in A_{g}$. Then $\left(A a_{g}\right)_{h g}=\left(A_{h} a_{g}\right)_{h g}=0$; it follows that $A \cdot a_{g} p_{(h g)^{-1}}=\left(A a_{g}\right)_{h g}=0$, which contradicts faithfulness. 
Conversely, assume the conditions, but say that $A \cdot x=0$, some $x \in A \# k[G]^{*}$. Write $x=\Sigma_{i} a_{i} p_{g_{i}}$, where $g_{i} \neq g_{j}$ when $i \neq j$, and all $a_{i} \neq 0$. Now, since $a_{j} \neq 0$, $\left(a_{j}\right)_{k} \neq 0$ for some $k \in G$. Let $h=g_{j}^{-1} k^{-1}$. Since $A \cdot x=0, A_{h} x=0=\sum_{i}\left(A_{h} a_{i}\right)_{g_{i}^{-1}}$. Since the $g_{i}$ are distinct, $\left(A_{h} a_{j}\right)_{g_{l}^{-1}}=0$. Since $h=g_{j}^{-1} k^{-1},\left(A_{h} a_{j}\right)_{g_{i}^{-1}}=A_{h}\left(a_{j}\right)_{k}=0$, a contradiction.

It is clear from the lemma that in the definition, $(3) \Rightarrow(2) \Rightarrow(1)$. To see that the three definitions are distinct, we consider some examples.

EXAMPLE 2.6. A nondegenerate grading which is not faithful.

This is essentially in [6]. Let $A=M_{2}(R)$, the $2 \times 2$ matrix ring over another ring $R$ with 1 , and let $G=\langle g\rangle$ be cyclic of order 3. Let $A_{1}=\left(\begin{array}{ll}R & 0 \\ 0 & R\end{array}\right), A_{g}=\left(\begin{array}{ll}0 & R \\ 0 & 0\end{array}\right)$, and $A_{g^{-1}}=\left(\begin{array}{cc}0 & 0 \\ R & 0\end{array}\right)$. Then $A_{g} A_{g^{-1}}=\left(\begin{array}{cc}R & 0 \\ 0 & 0\end{array}\right)$ and $A_{g^{-1}} A_{g}=\left(\begin{array}{cc}0 & 0 \\ 0 & R\end{array}\right)$, so the grading is nondegenerate; however $\left(\begin{array}{ll}0 & 0 \\ 0 & 1\end{array}\right) \cdot A_{g}=(0)$, so the grading is not faithful.

EXAMPLE 2.7. A faithful grading which is not strongly graded.

Let $A=\mathbf{Q}[x]$, the polynomials over the rationals, and let $G=\langle g\rangle$ have order 2 . Let $A_{1}=\mathbf{Q}\left[x^{2}\right]$ and let $A_{g}=\left\{\sum_{i} a_{i} x^{i} \mid i\right.$ odd $\}$. Then $A=A_{1} \oplus A_{g}$, and $A$ is faithfully graded since it is a domain. However, $1 \notin A_{g} A_{g}=A_{g} A_{g^{-1}}$, so $A$ is not strongly graded.

The various gradings relate to intersections of ideals.

LEMMA 2.8. (1) If the grading is nondegenerate and I is a right (left) ideal of $A$ with $I \cap A_{1}=0$, then $I \cap A_{g}=0$, all $g \in G$.

(2) If the grading is faithful and $I$ is a right (left) ideal of $A$ with $I \cap A_{h}=0$ for some $h \in G$, then $I \cap A_{g}=0$ for all $g \in G$.

Proof. (1) Let $I$ be a right ideal; a similar argument will work for left ideals. Now $\left(I \cap A_{g}\right) A_{g^{-1}} \subseteq I \cap A_{1}=(0)$, so $I \cap A_{g}=(0)$ by Lemma 2.5 (2) is very similar: $\left(I \cap A_{g}\right) A_{g^{-1} h} \subseteq I \cap A_{h}=(0)$, so $I \cap A_{g}=(0)$ by Lemma 2.5 .

The next result is the analog of the theorem of Fisher and Montgomery for group actions [11]. The graded ring $A$ is graded semiprime if it has no nonzero nilpotent graded ideals.

THEOREM 2.9. The following are equivalent:

(1) $A$ is graded semiprime.

(2) $A_{1}$ is semiprime and the grading is nondegenerate.

(3) $A \# k[G]^{*}$ is semiprime.

Proof. (1) $\Rightarrow(2)$ is just [6, Proposition 1.2(3)].

(3) $\Rightarrow$ (1) follows from the fact that if $I$ is a nilpotent graded ideal of $A$, it generates a nilpotent ideal of $A \# k[G]^{*}$.

It remains to show that (2) $\Rightarrow(3)$. Say that $A \# k[G]^{*}$ is not semiprime; then there exists $0 \neq x \in A \# k[G]^{*}$ so that $x\left(A \# k[G]^{*}\right) x=0$. Choose $p_{g}$ in the "support" of $x$ so that $x p_{g}=a p_{g} \neq 0, a \in A$. Then $a p_{g}\left(A \# k[G]^{*}\right) a p_{g}=0$, and consequently $0=$ $a p_{g}\left(p_{g} A\right) a p_{g}=a\left(p_{g} A a p_{g}\right)=a(A a)_{1} p_{g}$ by Corollary 1.5. Thus $A a(A a)_{1}=0$, and so $(A a)_{1}^{2}=0$. Since $A_{1}$ is semiprime, $(A a)_{1}=0$. Thus $(A, a)=0$, and so by nondegeneracy $a=0$, a contradiction.

We apply Theorem 2.9 to the prime radical in $\$ 5$.

A similar result holds for $A \# k[G]^{*}$ being prime. 
THEOREM 2.10. The following are equivalent:

(1) $A_{1}$ is prime and $A$ is graded semiprime.

(2) $A_{1}$ is prime and the grading is nondegenerate.

(3) $A_{1}$ is prime and the grading is faithful.

(4) $A \# k[G]^{*}$ is prime.

Proof. (1) $\Rightarrow$ (2) follows as in Theorem 2.9 by [6, Proposition 1.2]. For (2) $\Rightarrow(3)$, say that $a_{g} A_{h}=0$, for some $g, h \in G$, where $a_{g} \in A_{g}$. By nondegeneracy, $0 \neq$ $A_{h} A_{h^{-1}}=I$, a nonzero ideal of $A_{1}$, and $0 \neq A_{g^{-1}} a_{g} \subseteq A_{1}$. But $\left(A_{g^{-1}} a_{g}\right) I=(0)$, which contradicts $A_{1}$ being prime. Thus the grading is faithful.

(3) $\Rightarrow$ (4) follows by an argument similar to Theorem 2.9. That is, if $A \# k[G]^{*}$ is not prime, then there exist $0 \neq x, y \in A \# k[G]^{*}$ so that $x\left(A \# k[G]^{*}\right) y=0$. By choosing $p_{g}$ and $p_{h}$ in the "support" of $x$ and $y$, respectively, we may assume that $x=a p_{g}$ and $y=b p_{h}, a, b \in A$. It follows that $0=a p_{g}\left(A p_{h} A\right) b p_{h}=a A_{g^{-1} h}(A b)_{1} p_{h}$ $=0$, and so $a A_{g^{-1} h}(A b)_{1}=0$. Hence $A a A_{g^{-1} h}(A b)_{1} A_{h^{-1} g}=0$, and so $(A a)_{1} A_{g^{-1} h}(A b)_{1} A_{h^{-1} g}=0$. Now $A_{g^{-1} h}(A b)_{1} A_{h^{-1} g}$ is an ideal of $A_{1}$, and it is nonzero since $(A b)_{1} \neq 0$ by nondegeneracy and since by faithfulness, $A_{g^{-1} h}(A b)_{1} A_{h^{-1} g} \neq 0$ by Lemma 2.5. But then since $A_{2}$ is prime, $(A a)_{1}=0$. Thus $a=0$ by nondegeneracy, a contradiction.

Finally we show (4) $\Rightarrow(1)$. If $A \# k[G]^{*}$ is prime, certainly $A$ is graded semiprime as in Theorem 2.9. Moreover, by Corollary $1.5, A_{1} \cong p_{1}\left(A \# k[G]^{*}\right) p_{1}$, and so is a prime ring.

Another interpretation of Theorem 2.10 can be given in terms of Morita contexts. For any Morita context $\left[R,{ }_{R} V_{S},{ }_{S} W_{R}, S\right]$, one can form a ring

$$
C=\left(\begin{array}{ll}
R & V \\
W & S
\end{array}\right)
$$

The Morita context is called prime if $C$ is a prime ring.

Corollary 2.11. For $A$ graded by $G,\left[A_{1}, A, A, A \# k[G]^{*}\right]$ is a prime Morita context $\Leftrightarrow$ any of the conditions in Theorem 2.10 hold.

Proof. $(\Rightarrow)$ If $C$ is prime, let $e=\left(\begin{array}{ll}0 & 0 \\ 0 & 1\end{array}\right)$. Then also $e C e$ is prime, but $e C e \cong S=$ $A \# k[G]^{*}$, a prime ring, and all the conditions in the theorem are equivalent.

$(\Leftarrow)$ By the theorem, and Lemma 2.5, $A$ is faithful as a left and right $A \# k[G]^{*}$ module. Since the form [, ] is always nondegenerate, this implies that $[A \cdot s, A]=0$ implies $s=0$, for $s \in S$. Hence by [22, Proposition 3], the Morita context is prime.

We now characterize strongly $G$-graded rings in terms of the Morita context. The proof is based on an argument of M. Rieffel, who showed that if $G$ is an abelian group of automorphisms of an algebra $A$ over the complex numbers, then the skew group ring $A * G$ is Morita equivalent to $A^{G}$ if and only if $A$ is strongly $\hat{G}$-graded, where $\hat{G}$ is the dual group of $G$. We wish to thank him for making his argument available.

THEOREM 2.12. Let $A$ be a G-graded ring, and consider the Morita context in Proposition 2.4. Then $A \# k[G]^{*}$ is Morita equivalent to $A_{1}$ if and only if $A$ is strongly $G$-graded. 
Proof. $A \# k[G]^{*}$ is Morita equivalent to $A_{1}$ if and only if [, ] is onto; equivalently, if and only if $A p_{1} A=A \# k[G]^{*}$.

First assume that $A p_{1} A=A \# k[G]^{*}$. Then by Corollary 1.5,

$$
A_{1} p_{g}=p_{g}\left(A \# k[G]^{*}\right) p_{g}=\left(p_{g} A p_{1}\right)\left(p_{1} A p_{g}\right)=A_{g} A_{g^{-1}} p_{g} .
$$

It follows that $A_{1}=A_{g} A_{g^{-1}}$, all $g \in G$. That is, $A$ is strongly $G$-graded.

Conversely, assume $A_{g} A_{g^{-1}}=A_{1}$ for all $g \in G$. In order to show that $1 \in A p_{1} A$, it suffices to show that each $p_{g} \in A p_{1} A$, all $g \in G$. For a fixed $g, A_{g} A_{g^{-1}}=A_{1}$ implies that there exists $\left\{a_{i}\right\} \subset A_{g},\left\{b_{i}\right\} \subset A_{g^{-1}}$ such that $\sum_{i=1}^{n} a_{i} b_{i}=1$. Thus, using the fact that $\left(b_{i}\right)_{h^{-1}}=\delta_{g, h} b_{i}$,

$$
p_{g}=\sum_{i=1}^{n} a_{i} b_{i} p_{g}=\sum_{i} a_{i}\left(\sum_{h \in G}\left(b_{i}\right)_{h^{-1}} p_{h}\right)=\sum_{i=1}^{n} a_{i} p_{1} b_{i} \in A p_{1} A .
$$

The theorem is proved.

By combining Theorems 2.12 and 2.2, we obtain a result of Dade [7, Theorem 2.8]. Alternatively, Theorem 2.12 could have been proved using Theorem 2.2 and Dade's result.

Corollary 2.13. A is strongly G-graded if and only if there is a category equivalence between $\operatorname{Mod}\left(A_{1}\right)$ and $\operatorname{Gr} \operatorname{Mod}(A)$.

REMARK 2.14. Some of Fell's results in [9] can be interpreted as the $C^{*}$-algebra analogs of Theorems 2.2, 2.12 and Corollary 2.13. For let $B$ be a Banach *-algebraic bundle over the locally compact group $G$ (see Remark 1.6). The "systems of imprimitivity" for $B$ correspond to certain graded $B$-modules, and in Theorem 30.3, Fell gives a one-to-one correspondence between systems of imprimitivity for $B$ and certain representations of the transformation bundle $D$; this is analogous to our Theorem 2.2. In his Theorem 32.8, under the assumption that $B$ is saturated (strongly $G$-graded), he gives a correspondence between systems of imprimitivity for $B$ over $G / H$ and representations of $B_{H}$, for any closed subgroup $H$ of $G$, where $B_{H}$ is the part of $B$ over $H$. When $H=\langle 1\rangle$, this is the analog of Corollary 2.13. The more general result extends the classical imprimitivity theorem of Mackey.

3. The duality theorems. The two duality theorems in this section are already known to operator algebraists, for $A$ a von Neumann algebra and $G$ a locally compact group; they were proved independently by Landstad [14], Nakagami [19], and Stratila, Voiculescu and Zsido [27]. The two dual algebras used were $L^{\infty}(G)$, where we have used $k[G]^{*}$, and $\Re(G)$, the von Neumann algebra on $L^{2}(G)$ generated by the regular representation of $G$, where we have used $k[G]$. These results generalize the work of Takesaki on abelian groups [29]. An exposition of these results is given by Nakagami and Takesaki in [20]. For results on $C^{*}$-algebras, see [26]. We give here elementary algebraic proofs of the theorems for finite groups; two fundamental simplifications in our case are that a coaction of $G$ is just a grading, and that the "crossed product with respect to a coaction" is just our smash product algebra described in Proposition 1.4, as noted in Remark 1.6. 
The other crucial ingredient in our arguments is the next lemma, which appears in [23, Lemma 1.6, p. 228], and is in essence due to Clifford. We wish to thank D. S. Passman for suggesting this as a method of proof.

LEMMA 3.1. Let $W$ be a ring, and let $1=e_{1}+e_{2}+\cdots+e_{n}$ be a decomposition of 1 into a sum of orthogonal idempotents. Let $G$ be a subgroup of the group of units of $W$, and assume that $G$ permutes the set $\left\{e_{1}, \ldots, e_{n}\right\}$ transitively by conjugation. Then $W \cong M_{n}(T)$, where $T$ is the ring $T=e_{1} W e_{1}$.

We first consider the Duality Theorem for Actions. Let $G$ be a finite group of order $n$, and let $\alpha: G \rightarrow \operatorname{Aut}_{k}(S)$ be an action of $G$ on the ring $S$. Form the skew group ring $R=S * G$. Then $R$ is certainly $G$-graded, by letting $R_{g}=S g$. Thus as in $\S 1$ we may form $R \# k[G]^{*}$.

Theorem 3.2 (DuAltTy For ACtions). $(S * G) \# k[G]^{*} \cong M_{n}(S)$.

Proof. We first claim that $G$ acts transitively on the idempotents $\left\{p_{h} \mid h \in G\right\}$. Now for any $s \in S, s g \in(S * G)_{g}$, so by Proposition 1.4, $p_{h}(s g)=(s g) p_{g^{-1} h}$. Letting $s=1$, we have $p_{h} g=g p_{g^{-1} h}$, or $g^{-1} p_{h} g=p_{g^{-1} h}$. Thus Lemma 3.1 applies and the theorem will follow if we can show that $p_{1}\left((S * G) \# k[G]^{*}\right) p_{1} \cong S$.

Using that the $\left\{p_{g}\right\}$ are orthogonal idempotents, we see $p_{1}\left((S * G) \# k[G]^{*}\right) p_{1}=$ $p_{1}(S * G) p_{1}$. But by Corollary $1.5, p_{1}(S * G) p_{1}=(S * G)_{1} p_{1}=S p_{1}$, which is isomorphic to $S$. The theorem is proved.

We now consider coactions. Let $R$ be a ring graded by a group $G$ of order $n$, and consider $S=R \# k[G]^{*}$ as in $\S 1$.

LEMMA 3.3. An action of $G$ on $R \# k[G]^{*}$ is given by

$$
\left(r p_{h}\right)^{g}=r p_{h g}, \quad \text { for } r \in R, p_{h} \in k[G]^{*}, g \in G .
$$

Proof. It suffices to check that $(x y)^{g}=x^{g} y^{g}$, for $x=r p_{h}, y=s p_{k} \in R \# k[G]^{*}$. Now $(x y)^{g}=\left(r p_{h} s p_{k}\right)^{g}=\left(r s_{h k^{-1}} p_{k}\right)^{g}=r s_{h k^{-1}} p_{k g}=r s_{(h g)(k g)^{-1}} p_{k g}=r p_{h g} s p_{k g}=$ $x^{g} y^{g}$. Thus $x \rightarrow x^{g}$ is an automorphism.

Clearly, the action in Lemma 3.3 is related to the regular representation of $G$.

We may therefore form the skew group ring $S * G=\left(R \# k[G]^{*}\right) * G$, in which $g^{-1}\left(r p_{h}\right) g=\left(r p_{h}\right)^{g}=r p_{h g}$, and so $r p_{h} g=g r p_{h g}$, all $r \in R, h \in G$.

LEMMA 3.4. $p_{1}\left(\left(R \# k[G]^{*}\right) * G\right) p_{1}=\Sigma_{g} \oplus R_{g} g p_{1} \cong R$.

Proof. From the group action, $g p_{1}=p_{g^{-1}} g$, for any $g \in G$. Since the $\left\{p_{g}\right\}$ are orthogonal, it follows that $p_{1}\left(\left(R \# k[G]^{*}\right) * G\right) p_{1}=p_{1}\left(\Sigma_{g} R \# p_{g^{-1}} g\right)=\Sigma_{g} \oplus R_{g} p_{g^{-1}} g$ $=\Sigma_{g} \oplus R_{g} g p_{1}$, since $p_{1} R p_{g^{-1}}=R_{g} p_{g^{-1}}$ by Corollary 1.5. We claim that $\Sigma_{g} R_{g} g p_{1} \cong$ $R$. Any $r \in R$ may be written as $r=\sum_{g} r_{g}$, where $r_{g} \in R_{g}$; so define $\phi: R \rightarrow \Sigma_{g} R_{g} g p_{1}$ by $\phi(r)=\Sigma_{g} r_{g} g p_{1}$. $\phi$ is clearly an isomorphism of abelian groups. To show it preserves multiplication, it suffices to show $\phi\left(r_{g} r_{h}\right)=\phi\left(r_{g}\right) \cdot \phi\left(r_{h}\right)$, for $r_{g} \in R_{g}$, $r_{h} \in R_{h}$. Now $\phi\left(r_{g}\right) \phi\left(r_{h}\right)=\left(r_{g} g p_{1}\right)\left(r_{h} h p_{1}\right)=r_{g} g\left(p_{1} r_{h} p_{h^{-1}}\right) h=r_{g} g r_{h} p_{h^{-1}} h=$ $r_{g} r_{h}(g h) p_{1}=\phi\left(r_{g} r_{h}\right)$, since $r_{g} r_{h} \in R_{g h}$. The lemma is proved.

Theorem 3.5 (Duality for CoActions). $\left(R \# k[G]^{*}\right) * G \cong M_{n}(R)$. 
Proof. It is clear from the action of $G$ on $R \# k[G]^{*}$ that $G$ permutes the orthogonal idempotents $\left\{p_{g}\right\}$. Thus the theorem follows immediately from Lemmas 3.1 and 3.4 .

A natural question to ask at this point is the following: for what other finitedimensional Hopf algebras $H$, with dual Hopf algebra $H^{*}$, do the analogs of Theorems 3.2 and 3.5 hold? That is, when is $(A \# H) \# H^{*} \cong M_{n}(A)$ ?

4. Jacobson radicals and a question of Bergman. Returning to a $k$-algebra $A$ graded by $G$, we compare the Jacobson radicals of $A \# k[G]^{*}$ and $A_{1}$, and the graded Jacobson radical of $A$; in doing so we answer the question of Bergman mentioned in the introduction.

As in [3], the graded Jacobson radical $J_{G}(A)$ is defined to be the intersection of all annihilators of graded irreducible right $A$-modules. By standard arguments, $J_{G}(A)$ is also the intersection of the maximal graded right ideals, and the definition is left-right symmetric.

We first consider $A \# k[G]^{*}$.

THEOREM 4.1. $J\left(A \# k[G]^{*}\right)=J_{G} \# k[G]^{*}$.

Proof. First, choose any $x \in J\left(A \# k[G]^{*}\right)$, say $x=\sum_{i} a_{i} p_{g_{i}}$, for $a_{i} \in A$, and let $V$ be any graded irreducible $A$-module. By Lemma $2.1, V$ is an $A \# k[G]^{*}$-module, and it is certainly irreducible as an $A \# k[G]^{*}$-module since it is irreducible as a graded $A$-module. Thus $V \cdot x=0$. We claim $V a_{i}=0$, all $i$ (and so $a_{i} \in J_{G}(A)$ and $x \in$ $\left.J_{G}(A) \# k[G]^{*}\right)$. Since $J=J\left(A \# k[G]^{*}\right)$ is an ideal, $x p_{g_{i}}=a_{i} p_{g_{i}} \in J$. Using the group action in Lemma 3.3, since $J$ is certainly $G$-stable, $a_{i} p_{g_{h} h} \in J$, all $h \in G$. Thus $a_{i} \cdot 1=a_{i}\left(\Sigma_{h} p_{g_{i} h}\right) \in J$, so $V a_{i}=0$, proving the claim. Thus $J\left(A \# k[G]^{*}\right) \subseteq$ $J_{G}(A) \# k[G]^{*}$.

Conversely, let $W$ be an irreducible $A \# k[G]^{*}$-module. By Lemma 2.1, $W$ is a graded $A$-module, by setting $W_{g}=W p_{g^{-1}}$. Then $W$ is irreduible as a graded $A$-module, since for any graded submodule $V=\Sigma_{g} \oplus V_{g}$, we have $V p_{h}=\Sigma_{g} \oplus V_{g} p_{h}=$ $V_{h^{-1}} \subseteq V$, and so $V$ is an $A \# k[G]^{*}$-submodule. Thus $W J_{G}(A)=0$. Since $J_{G}(A)$ annihilates all such $W, J_{G}(A) \subseteq J\left(A \# k[G]^{*}\right)$. The theorem is proved.

The fact that $J_{G}(A) \# k[G]^{*} \subseteq J\left(A \# k[G]^{*}\right)$ could have been obtained from known results. For, if $H$ is any Hopf algebra and $A$ any $H$-module algebra, a Jacobson-radical type constuction is given by $\mathrm{J}$. R. Fisher in [10]. Where $\mathscr{f}(A)$ is the analog of our $J_{G}(A)$, he proves that $\mathscr{f}(A) \# H \subseteq J(A \# H)$. The other containment is open in general.

Corollary 4.2. If $A$ is graded by the finite group $G$, then $J\left(A_{1}\right)=J_{G}(A) \cap A_{1}$.

Proof. For any ring $S$ with idempotent $e \in S$, it is well known that $J(e S e)=$ $e J(S) e$ [12]. Applying this with $S=A \# k[G]^{*}$ and $e=p_{1}$, and the fact that $p_{1} S p_{1}=A_{1} p_{1}$ (Corollary 1.5), we see that

$$
\begin{aligned}
J\left(A_{1}\right) p_{1} & =J\left(A_{1} p_{1}\right)=p_{1} J(S) p_{1}=p_{1}\left(J_{G}(A) \# k[G]^{*}\right) p_{1} \\
& =J_{G}(A)_{1} p_{1}=\left(J_{G}(A) \cap A_{1}\right) p_{1} .
\end{aligned}
$$

Thus $J\left(A_{1}\right)=J_{G}(A) \cap A_{1}$. 
We now consider $J(A)$. We use the following result of Villamayor [23, Chapter 7, Theorems 27, 31]. Although proved for group rings, the same arguments work for skew group rings [25, Theorem 7.1].

Proposition 4.3. Let $G$ act on the ring $R$. Then $J(R) * G \subseteq J(R * G)$, with equality if $|G|^{-1} \in R$. Moreover $J(R * G)^{|G|} \subseteq J(R) * G$.

We now answer Bergman's question, mentioned in the Introduction, by showing that $J_{G}(A) \subseteq J(A)$. Bergman had shown that it was true if $G$ was solvable [3]. The question was also answered in the special case that $A$ was strongly $G$-graded in [21].

THEOREM 4.4. Let $A$ be graded by the finite group $G$. Then

(1) $J_{G}(A) \subseteq J(A)$; in fact $J_{G}(A)=J(A)_{G}$,

(2) $J(A)^{|G|} \subseteq J_{G}(A)$,

(3) if $|G|^{-1} \in A$, then $J_{G}(A)=J(A)$.

Proof. Consider $\left(A \# k[G]^{*}\right) * G$, with the group action as in Lemma 3.3. Then by Theorem 4.1 and Proposition 4.3, $J\left(\left(A \# k[G]^{*}\right) * G\right) \supseteq J\left(A \# k[G]^{*}\right) * G=$ $\left(J_{G}(A) \# k[G]^{*}\right) * G$, with equality if $|G|^{-1} \in A$. Thus $J\left(p_{1}\left(\left(A \# k[G]^{*}\right) * G\right) p_{1}\right)=$ $p_{1} J\left(\left(A \# k[G]^{*}\right) * G\right) p_{1} \supseteq p_{1}\left(\left(J_{G}(A) \# k[G]^{*}\right) * G\right) p_{1}$. Using Lemma 3.4, $J\left(\Sigma_{g} A_{g} g p_{1}\right)$ $\supseteq \Sigma_{g}\left(J_{G}(A)\right)_{g} g p_{1}$. Since $\Sigma_{g} A_{g} g p_{1} \cong A$, it follows that $J(A) \supseteq J_{G}(A)$. But as Bergman notes in [3, Definition 11], $J_{G}(A)$ is the largest graded ideal $I$ of $A$ such that $I \cap A_{1}$ is a quasi-regular ideal of $A_{1}$. Thus $J(A)_{G} \subseteq J_{G}(A)$, proving (1). Since the containments are equalities if $|G|^{-1} \in R$, (3) follows also.

For (2), use the fact from Proposition 4.3 that $J\left(\left(A \# k[G]^{*}\right) * G\right)^{|G|} \subseteq$ $J\left(A \# k[G]^{*}\right) * G=\left(J_{G}(A) \# k[G]^{*}\right) * G$. Since for any ideal $I,\left(p_{1} I p_{1}\right)^{|G|} \subseteq p_{1} I^{|G|} p_{1}$, the result follows.

A consequence of the theorem is that $J_{G}(A)$ is a quasi-regular ideal of $A$. We can therefore obtain as a corollary the graded version of a theorem of Amitsur on polynomial rings. If $A$ is graded by $G$, then $A[x]$ is also graded by $G$ if we use $(A[x])_{g}=A_{g}[x]$.

Corollary 4.5. If $A$ has no nil graded ideals, then $J_{G}(A[x])=0$.

Proof. Follow the proof in [12, p. 150], with appropriate adjustments for graded rings.

5. Prime radicals. In this section we compare the prime radicals of $A, A \# k[G]^{*}$, and $A_{1}$, as was done in $\S 4$ for the Jacobson radical. These results are analogs of results known for groups acting on rings [17]. The prime radical of $A$ will be denoted $N(A)$.

For $G$ acting on a ring $S$, an ideal $\mathscr{G}$ is $G$-prime if whenever $\mathscr{A} \mathscr{B} \subseteq \mathcal{G}$, where $\mathcal{Q}, \mathscr{G}$ are $G$-stable ideals of $S$, then $\mathscr{Q} \subseteq \mathscr{9}$ or $\mathscr{B} \subseteq 9$. Equivalently, $\mathscr{G}=\cap_{g}{ }^{\mathcal{P} g}$, where $\mathscr{P}$ is a prime ideal of $S$ [15].

Analogously, for $A$ a graded ring, a graded ideal $I$ is graded prime if whenever $J K \subseteq I$, for $J, K$ graded ideals of $A$, then $J \subseteq I$ or $K \subseteq I$. The graded prime radical $N_{G}(A)$ is the intersection of all graded prime ideals of $A$. 
LEMma 5.1. If $A$ is any graded ring and I a graded ideal of $A$, then $I$ is a graded prime $\Leftrightarrow I=P_{G}$, the associated graded ideal of some prime $P$ of $A$.

Consequently $N_{G}(A)=N(A)_{G}$, the associated graded ideal of $N(A)$.

Proof. It is trivial that if $P$ is prime, then $P_{G}$ is a graded prime. Conversely, say that $I$ is a graded prime. Let $\delta$ be the set of all ideals $J$ of $R$ so that $J_{G}=I$; we may apply Zorn's lemma to $\delta$ and choose a maximal such ideal, call it $P$. Say that $K \supseteq P$, $L \supseteq P$ are ideals with $K L \subseteq P$, then $K_{G} L_{G} \subseteq P$, so either $K_{G} \subseteq P_{G}$ or $L_{G} \subseteq P_{G}$ since $P_{G}=I$ is a graded prime. By the maximality of $P$, it follows that $K \subseteq P$ or $L \subseteq P$. Thus $P$ is a prime of $A$.

The prime radical $N(A)$ can also be characterized as an ascending union $\left\{N_{\alpha}\right\}$ of ideals as follows: if $\alpha$ is not a limit ordinal, $N_{\alpha}$ is the sum of all ideals of $A$ which are nilpotent $\bmod N_{\alpha-1}$; if $\alpha$ is a limit ordinal, $N_{\alpha}=\cup_{\beta<\alpha} N_{\beta}$. For $A$ graded by $G$, we make the analogous definitions: $\left(N_{G}\right)_{\alpha}=\cup_{\beta<\alpha}\left(N_{G}\right)_{\beta}$ if $\alpha$ is a limit ordinal, and $\left(N_{G}\right)_{\alpha}$ is the sum of all graded ideals of $A$ which are nilpotent $\bmod \left(N_{G}\right)_{\alpha-1}$ when $\alpha$ is not a limit ordinal.

LEMMA 5.2. For all $\alpha,\left(N_{G}\right)_{\alpha}=\left(N_{\alpha}\right)_{G}$, and so $N_{G}(A)=\cup_{\alpha}\left(N_{G}\right)_{\alpha}$.

Proof. We proceed by induction on $\alpha$. If $\alpha$ is a limit ordinal, the assertion is trivial, so assume $\alpha$ has a predecessor $\alpha-1$. By induction $\left(N_{G}\right)_{\alpha-1}=\left(N_{\alpha-1}\right)_{G}$. Since $\left(N_{\alpha}\right)_{G}$ is a sum of ideals nilpotent mod $\left(N_{G}\right)_{\alpha-1}$, clearly $\left(N_{G}\right)_{\alpha} \subseteq\left(N_{\alpha}\right)_{G}$. Conversely, choose any $x \in\left(N_{\alpha}\right)_{G}$. Since $x$ is in a graded ideal, we may assume that $x$ is a homogeneous element. Now $x \in M$, where $M^{k} \subseteq N_{\alpha-1}$; but then $(A x A)^{k}$ is a graded ideal, so $(A x A)^{k} \subseteq\left(N_{\alpha-1}\right)_{G}=\left(N_{G}\right)_{\alpha-1}$. Thus $x \in\left(N_{G}\right)_{\alpha}$, proving the lemma.

TheOrem 5.3. $N\left(A \# k[G]^{*}\right)=N_{G}(A) \# k[G]^{*}=N(A)_{G} \# k[G]^{*}$.

Proof. By Theorem 2.9, $A \# k[G]^{*} / N_{G}(A) \# k[G]^{*} \cong\left(A / N_{G}(A)\right) \# k[G]^{*}$ is semiprime, and so $N\left(A \# k[G]^{*}\right) \subseteq N_{G}(A) \# k[G]^{*}$.

Conversely, by Lemma 5.2, it suffices to show that $\left(N_{G}\right)_{\alpha} \# k[G]^{*} \subseteq N\left(A \# k[G]^{*}\right)$, for each $\alpha$. But if $\alpha$ is not a limit ordinal, $\left(N_{G}\right)_{\alpha-1} \# k[G]^{*} \subseteq N\left(A \# k[G]^{*}\right)$ by induction, and $\left(N_{G}\right)_{\alpha} \# k[G]^{*}$ is a sum of nilpotent ideals $\bmod \left(N_{G}\right)_{\alpha-1} \# k[G]^{*}$. Thus $\left(N_{G}\right)_{\alpha} \# k[G]^{*} \subseteq N\left(A \# k[G]^{*}\right)$. The case of a limit ordinal is trivial.

We note that another proof of Theorem 5.3 could be given using Theorem 6.2. However, the present proof is shorter, and Lemma 5.2 is of some interest in its own right.

Corollary 5.4. $N\left(A_{1}\right)=N_{G}(A) \cap A_{1}=N(A) \cap A_{1}$.

Proof. This follows from the theorem, using the same argument as in Corollary 4.2, and the fact that $N(A) \cap A_{1}=N_{G}(A) \cap A_{1}$.

Corollary 5.5. Assume that $A$ has no $|G|$-torsion.

(1) $N_{G}(A)=N(A)$.

(2) If $A_{1}$ is semiprime and the grading is nondegenerate, then $A$ is semiprime. 
Proof. (1) It is known that $A / N(A)$ has no $|G|$-torsion [17, Lemma 1.8] and it follows that $A / N(A)_{G}$ has no $|G|$-torsion; that is, we may assume that $A$ is graded semiprime, and we wish to show it is semiprime. Now $A \# k[G]^{*}$ is semiprime by Theorem 2.9; using the action of $G$ on $A \# k[G]^{*}$ (Lemma 3.3) we may consider the skew group ring $\left(A \# k[G]^{*}\right) * G$, which is semiprime by Fisher and Montgomery [11]. But the Duality Theorem for Coactions gives $\left(A \# k[G]^{*}\right) * G \cong M_{n}(A)$. Thus $A$ is semiprime.

(2) By Theorem 2.9, the hypotheses imply that $A$ is graded semiprime. Thus $A$ is semiprime by (1).

We remark that Corollary 5.5 was proved in [21] in the special case that $A$ is strongly $G$-graded.

6. Prime ideals of $A$ and $A \# k[G]^{*}$. In this section, we compare the prime ideals of $A$ and the prime ideals of $A \# k[G]^{*}$; these results are analogs of the theorems of Lorenz and Passman on crossed products [15]. As a consequence, we obtain an incomparability theorem for primes of the ring extension $A_{1} \subseteq A$.

Our method of proof is to use the Duality Theorem for Coactions and reduce the problem to Lorenz and Passman's theorems; thus we must examine more carefully the action of $G$ on $A \# k[G]^{*}$. Recall from $\S 3$ that this is given as follows: for $a \in A$, $p_{h} \in k[G]^{*}, g \in G$,

$$
\left(a p_{h}\right)^{g}=a p_{h g} .
$$

Note that the fixed ring $(A \# k[G])^{G}=A \cdot 1=A$.

Lemma 6.1. Let 9 be an ideal of $A \# k[G]^{*}$. Then

(1) $9 \cap A=\left(\cap_{g} g^{g}\right) \cap A$,

(2) $I \cap A$ is a graded ideal of $A$,

(3) if 9 is also $G$-stable, then $9=(9 \cap A) \# k[G]^{*}$. In particular, $9 \cap A \neq 0$ if $9 \neq 0$.

Proof. (1) Since $9 \cap A$ is just the set of fixed elements in 9 , certainly $9 \cap A=$ $g^{g} \cap A$, all $g \in G$, and so $\left(\cap_{g} g^{g}\right) \cap A=\cap_{g}\left(g^{g} \cap A\right)=9 \cap A$.

(2) Say that $a=a \cdot 1 \in \mathscr{9} \cap A$. For any $h \in G, p_{h} a=\sum_{g} a_{h g^{-1}} p_{g} \in \mathscr{G}$, using Proposition 1.4. Since the $\left\{p_{g}\right\}$ are orthogonal, $p_{h} a p_{k}=a_{h k^{-1}} p_{k} \in \mathcal{G}$ for $k \in G$. Since both $h$ and $k$ are arbitrary, this mean that $a_{g} p_{h} \in \mathcal{G}$, all $g, h \in G$. But then $a_{g}=a_{g} \cdot 1=a_{g}\left(\Sigma_{h} p_{h}\right) \in 9$. Thus $9 \cap A$ is graded.

(3) Now assume that $\mathscr{G}$ is also $G$-stable, and choose $x=\sum_{i} a_{i} p_{g_{i}} \in \mathscr{G}$ where $g_{i} \neq g_{j}$ if $i \neq j$. It suffices to show that $a_{i} \in \mathcal{G} \cap A$, for all $i$. Now $x p_{g_{i}}=a_{i} p_{g_{i}} \in \mathcal{G}$, and so $\Sigma_{h \in G}\left(a_{i} p_{g_{i}}\right)^{h}=a_{i}\left(\Sigma_{h} p_{g_{i} h}\right)=a_{i} \cdot 1 \in \mathscr{G} \cap A$. The lemma is proved.

We can prove the first main result of this section.

THEOREM 6.2. Consider $A \# k[G]^{*}$, where $A$ is graded by $G$.

(1) If $P$ is a prime ideal of $A$, then there exists a prime 9 of $A \# k[G]^{*}$ so that $\mathscr{P} \cap A=P_{G} . \mathscr{P}$ is unique up to its $G$-orbit $\left\{\mathscr{\rho}^{g}\right\}$, and $P_{G} \# k[G]^{*}=\cap_{g}{ }^{\rho g}$, a $G$-prime ideal of $A \# k[G]^{*}$.

(2) If $\mathcal{P}$ is any prime ideal of $A \# k[G]^{*}$, then $\mathcal{P} \cap A=P_{G}$, for some prime $P$ of $A$, and (1) applies. 
Proof. (1) Since $P$ is prime, $P_{G}$ is a graded prime ideal of $A$ by Lemma 5.1. Then $P_{G} \# k[G]^{*}$ is a $G$-prime ideal of $A \# k[G]^{*}$, for if $\Theta, 96$ are $G$-stable ideals of $A \# k[G]^{*}$ with $(中 ⿰ \cap) \subseteq P_{G} \# k[G]$, then $(\Theta \cap A)(9 \cap \cap A) \subseteq P_{G}$. Since by Lemma 6.1, (') $\cap A$ and $\because \cap \cap A$ are graded ideals of $A$, either $\mathcal{A}^{\prime} \cap A \subseteq P_{G}$ or $\cap \cap A \subseteq P_{G}$. Say that $\|^{\prime} \cap A \subseteq P_{G}$. Then by Lemma 6.1, $\Theta^{*}=\left(\psi^{\prime} \cap A\right) \# k[G]^{*} \subseteq P_{G} \# k[G]^{*}$. Since it is a $G$-prime ideal, there exists a prime ideal of of $A \# k[G]^{*}$ so that $P_{G} \# k[G]^{*}=$ $\cap_{g}(\rho) g$. Certainly $\left(\cap_{g}(\rho g) \cap A=P_{G}\right.$, and by Lemma 6.1, this is just $\odot \cap \cap$. Finally, the uniqueness part: let $\mathcal{Q}$ be another prime of $A \# k[G]^{*}$ with $\mathcal{Q} \cap A=P_{G}$. Then $\cap_{g}{ }^{{ }^{2}}{ }^{g}$ is a $G$-stable ideal with $\left(\cap_{g}{ }^{2}{ }^{g}\right) \cap A=P_{G}$, so by Lemma 6.1, $\cap_{g}{ }^{g}{ }^{g}=$ $P_{G} \# k[G]^{*}=\bigcap_{g}{ }^{(p g} g$. But now since $G$ is finite and the $\left\{\mathcal{Q}^{g}\right\},\left\{P^{g} g\right.$, are all primes, it follows that $\stackrel{Q}{\nu}=\left(\mathrm{P}^{h}\right.$, for some $h \in G$.

(2) Let $\mathscr{P}$ be any prime ideal of $A \# k[G]^{*}$. Then $\mathscr{P} \cap A$ is a graded ideal of $A$ by Lemma 6.1; we claim it is a graded prime. For if $I, J$ are graded ideals of $A$ with $I J \subseteq \mathscr{P} \cap A$, then $I \# k[G]^{*}$ and $J \# k[G]^{*}$ are ideals of $A \# k[G]^{*}$ whose product is in $\mathscr{P}$; as $\mathscr{P}$ is prime, one of them, say $I \# k[G]^{*} \subseteq \mathscr{P}$. But then $I \subseteq \mathscr{P} \cap A$, proving the claim. Now by Lemma 5.1, there exists a prime $P$ of $A$ with $P_{G}=\mathscr{\rho} \cap A$. The theorem is proved.

We note that an easy consequence of Theorem 6.2 is that $A$ is a graded prime ring $\Leftrightarrow A \# k[G]^{*}$ is $G$-prime. This would provide an alternate proof of Theorem 5.3.

We now turn to primes of $A$. Again duality is the key to the argument, reducing the problem to Lorenz and Passman's theorem [15, Theorem 1.3].

THEOREM 6.3. Let $A$ be graded by the finite group $G$, and let $Q$ be a graded prime ideal of $A$.

(1) $A$ prime ideal $P$ of $A$ is minimal over $Q \Leftrightarrow P_{G}=Q$.

(2) There are finitely many such minimal primes, say $P_{1}, \ldots, P_{m}$, and $m \leqslant|G|$.

(3) If $I=P_{1} \cap \cdots \cap P_{m}$, then $I^{|G|} \subseteq Q$, and $I=Q$ if $A / Q$ has no $|G|$-torsion.

Proof. By passing to the ring $A / Q$, we may assume that $A$ is a graded prime ring and that $Q=(0)$. Letting $R=A \# k[G]^{*}$, which is a $G$-prime ring by Theorem 6.2 , we apply Lorenz and Passman's theorem to $R * G=\left(A \# k[G]^{*}\right) * G$ to see the following: (1) a prime ideal $P^{\prime}$ of $R * G$ is minimal $\Leftrightarrow P^{\prime} \cap R=0$; (2) there are $m \leqslant|G|$ such minimal primes, call them $P_{1}^{\prime}, P_{2}^{\prime}, \ldots, P_{m}^{\prime}$; and (3) $I^{\prime}=P_{1}^{\prime} \cap \cdots \cap P_{m}^{\prime}$ is the unique largest nilpotent ideal of $R * G$ and $\left(I^{\prime}\right)^{|G|}=(0)$.

But the Duality Theorem for Coactions gives that $R * G \cong M_{n}(A)$; since for any ideal $J^{\prime}$ of $M_{n}(A), J^{\prime}=M_{n}(J)$ where $J$ is an ideal of $A$, there is a one-to-one correspondence $\phi: J^{\prime} \rightarrow J$, preserving intersections and inclusions, between ideals of $\left(A \# k[G]^{*}\right) * G$ and ideals of $A$. Thus there are $m \leqslant|G|$ minimal primes of $A$, say $P_{1}, \ldots, P_{m}$, and if $I=P_{1} \cap \cdots \cap P_{m}$, then $I^{|G|}=(0)$. By Corollary 5.4, when $A$ has no $|G|$-torsion, $A$ is semiprime, and so $I=0=Q$. It remains only to show (1): $P$ is a minimal prime if and only if $P_{G}=(0)$.

To see this, we must examine the Duality Theorem more carefully. From Lemma 3.4 , the copy of $A$ appearing in $M_{n}(A)$ is actually

$$
p_{1}\left(\left(A \# k[G]^{*}\right) * G\right) p_{1}=\Sigma_{g} \oplus A_{g} g p_{1} \cong A \text {. }
$$


From above, the prime $P^{\prime}$ is minimal in $R * G \Leftrightarrow P^{\prime} \cap R=0$. Using $\phi$, we have $P$ is minimal in $A \Leftrightarrow \phi\left(P^{\prime}\right) \cap \phi(R)=0 \Leftrightarrow p_{1} P^{\prime} p_{1} \cap p_{1}\left(A \# k[G]^{*}\right) p_{1}=0 \Leftrightarrow p_{1} P^{\prime} p_{1} \cap$ $A_{1} p_{1}=0$, by Corollary 1.5. Thus, $P$ is minimal in $A \Leftrightarrow P \cap A_{1}=0$. We now apply Lemma 2.8 to see that $P \cap A_{g}=0$ for all $g \in G$; that is $P_{G}=(0)$. The theorem is proved.

As a corollary, we may extend Corollary 5.5(1), to the case when $|G|$-torsion is allowed.

Corollary 6.4. $N(A)^{|G|} \subseteq N_{G}(A)$.

Proof. For any graded prime $Q$ of $A$, let $I=P_{1} \cap \cdots \cap P_{m}$ as in Theorem 6.3, where for each $P_{i},\left(P_{i}\right)_{G}=Q$. Since $N(A) \subseteq I, N(A)^{|G|} \subseteq Q$. Since this is true for all such $Q, N(A)^{|G|} \subseteq \cap Q=N_{G}(A)$.

7. Prime ideals of $A$ and $A_{1}$. In this last section, we compare the prime ideals of $A$ and $A_{1}$. Our first main theorem, on incomparability of primes, follows directly from the results of $\S 6$. As mentioned in the Introduction, it generalizes Lorenz and Passman's theorem on incomparability of primes in the extension $R \subseteq R * G$, where $R * G$ is a crossed product.

THEOREM 7.1. Let $A$ be graded by the finite group $G$. If $P \subsetneq Q$ are prime ideals of $A$, then $P \cap A_{1} \subsetneq Q \cap A_{1}$.

Proof. By passing to $A / P_{G}$, we may assume $A$ is graded and $P_{G}=0$. But then $Q$ is not a minimal prime, and so $Q_{G} \neq 0$ by Theorem 6.3. Thus $Q \cap A_{1} \neq 0$ by Lemma 2.8.

We now turn to the question of primes of $A$ lying over those of $A_{1}$, and conversely. The idea is analogous to that used to study primes of $R$ and $R^{G}$, when $|G|^{-1} \in R$. However, no characteristic assumptions are required here. We summarize what is needed about a ring extension $f S f \subset S$, where $f$ is a nonzero idempotent of the ring $S$. Although it is well known, a very clear proof appears in [15, Lemma 4.5]. Let $\operatorname{Spec}_{f}(S)$ denote the set of prime ideals of $S$ not containing $f$, and let $\operatorname{Spec}(f S f)$ be the set of primes of $f S f$.

LEMMA 7.2. Let $f$ be a nonzero idempotent of the ring $S$. Then the map $\psi$ : $P \rightarrow f P f=P \cap f S f$ sets up a one-to-one correspondence between $\operatorname{Spec}_{f}(S)$ and Spec( $f S f)$. Moreover, for $P, P_{1}, P_{2}$ prime ideals of $S, P_{1}^{\psi} \subset P_{2}^{\psi}$ if and only if $P_{1} \subset P_{2}$, and $P^{\psi}$ is primitive if and only if $P$ is primitive.

We shall use $S=A \# k[G]^{*}$ and $f=p_{1}$; by Corollary 1.5, $f S f=A_{1} p_{1} \cong A_{1}$. Thus there is a one-to-one correspondence between $\operatorname{Spec}_{p_{1}}\left(A \# k[G]^{*}\right)$ and $\operatorname{Spec}\left(A_{1}\right)$. Our fundamental theorem is the following:

THEOREM 7.3. Let $A$ be graded by the finite group $G$.

(1) If $P$ is any prime ideal of $A$, then there are $k \leqslant|G|$ primes $p_{1}, \ldots, p_{k}$ of $A_{1}$ minimal over $P \cap A_{1}$, and moreover $P \cap A_{1}=p_{1} \cap \cdots \cap p_{k}$. The set $\left\{p_{1}, \ldots, p_{k}\right\}$ is uniquely determined by $P$. 
(2) Given any prime $p$ of $A_{1}$, there exists a prime $P$ of $A$ so that $p$ is minimal over $P \cap A_{1}$. There are at most $m \leqslant|G|$ such primes $P_{1}, \ldots, P_{m}$ of $A$; they are precisely those primes satisfying $\left(P_{i}\right)_{G}=P_{G}$.

Proof. (1) Since $P \cap A_{1}=P_{G} \cap A_{1}$, we may pass to the graded prime ring $A / P_{G}$, and so it suffices to prove the result when $0=P_{G}=P \cap A_{1}$. By Theorem 6.2(1), there exists a prime $\mathscr{P}$ of $A \# k[G]^{*}$ so that $\mathscr{P} \cap A=(0)$, and $\cap_{g} \mathcal{P g}=(0)$. $\mathscr{P}$ is unique up to its $G$-orbit $\left\{\mathscr{P}^{g}\right\}$. Now apply $\psi$ in Lemma $7.2 ; \psi: \operatorname{Spec}_{p_{1}}\left(A \# k[G]^{*}\right) \rightarrow$ $\operatorname{Spec}\left(A_{1}\right)$. Thus $\cap_{g}\left(\rho^{g}\right)^{\psi}=(0) \cap A_{1}$. Letting $p_{i}=\left(\rho g_{i}\right)^{\psi}$, we have $p_{1} \cap \cdots \cap p_{n}=$ (0); throwing away any which are redundant, we have the desired set of $m \leqslant|G|$ minimal primes of $A_{1}$. The uniqueness of the $G$-orbit $\left\{\rho^{g}\right\}$ determines the $\left\{p_{i}\right\}$.

(2) Now consider a prime $p$ of $A_{1} ; p=\rho^{\psi}$ for some prime $\mathscr{\rho}$ of $A \# k[G]^{*}$. By Theorem 6.2(2), $\mathcal{P} \cap A=P_{G}$, for some prime $P$ of $A$. By Theorem 6.3, there are finitely may such primes $P_{1}, \ldots, P_{m}$, with $m \leqslant|G|$, so that $\left(P_{i}\right)_{G}=P_{G}=\mathscr{P} \cap A$; they are the primes of $A$ minimal over $P_{G}$. Applying (1), the primes of $A_{1}$ which are minimal over $P \cap A_{1}=P_{G} \cap A_{1}$ are precisely the set $\left\{\left(\rho^{g}\right)^{\psi}\right\}$. Since $p$ is in this set, $p$ is minimal over $P \cap A$.

As our last topic, we improve the result on Goldie rank obtained by Cohen and Rowen in [6]. We will show that the Joseph and Small "additivity principle" [13] holds between primes of $A$ and $A_{1}$, by an argument similar to that used for fixed rings in [16].

We denote the Goldie rank of a $\operatorname{ring} R$ by $\operatorname{rk}(A)$; if $R$ is graded by $G, \operatorname{rk}_{G}(R)$ is the graded Goldie rank of $R$. The following proposition is due to Cohen and Rowen [6, Propositions 1.5, 1.6, Theorem 1.7], suitably restated.

Proposition 7.4. Assume that $A$ is graded semiprime.

(1) $\operatorname{rk}\left(A_{1}\right)$ is finite $\Leftrightarrow \operatorname{rk}_{G}(A)$ is finite; in that case $\operatorname{rk}\left(A_{1}\right) \leqslant \operatorname{rk}_{G}(A) \leqslant|G| \operatorname{rk}(A)$.

(2) $A_{1}$ is Goldie $\Leftrightarrow A$ is graded Goldie $\Leftrightarrow A$ is Goldie.

(3) If (2) holds, then $A$ has an Artinian classical quotient ring $Q(A)=A_{T}$, where $T=\left\{\right.$ regular elements in $\left.A_{1}\right\}$.

We also recall the additivity principle.

Proposition 7.5 [13, Lemma 3.8]. Let $R \subset S$ be Artinian rings with the same 1, let $P$ be a prime ideal of $S$, and let $Q_{1}, \ldots, Q_{r}$ be the primes of $R$ minimal over $P \cap R$. Then there exist positive integers $z_{1}, \ldots, z_{r}$ such that

$$
\operatorname{rk}(S / P)=\sum_{i=1}^{r} z_{i} \operatorname{rk}\left(R / Q_{i}\right) .
$$

We can now prove our theorem.

THeOREM 7.6. Let $A$ be graded by $G$, and let $P$ be any prime of $A$ such that $A / P_{G}$ is a graded Goldie ring. Let $p_{1}, \ldots, p_{k}$ be the primes of $A_{1}$ which are minimal over $P \cap A_{1}$, given by Theorems 7.2. Then $A_{1} / p_{i}$ is Goldie, for all $i$, and there exist positive integers $z_{1}, \ldots, z_{k}$ such that

$$
\operatorname{rk}(A / P)=\sum_{i=1}^{k} z_{i} \operatorname{rk}\left(A_{1} / p_{i}\right)
$$


Proof. By passing to the ring $A / P_{g}$, we may assume that $A$ is a graded prime ring which is graded Goldie, and $P$ is a prime ideal with $P_{G}=(0)=P \cap A_{1}=$ $p_{1} \cap \cdots \cap p_{k}$. By Proposition 7.4, $A$ is Goldie, with an Artinian quotient ring $S=Q(A)=A_{T}$, where $T$ is the set of regular elements of $A_{1}$. Also $A_{1}$ is Goldie, and semiprime; since $p_{1}$ is a minimal prime of $A_{1}, A_{1} / p_{i}$ is Goldie, all $i$. Now let $R=Q\left(A_{1}\right)=\left(A_{1}\right)_{T} ; R$ is also Artinian, so we may apply Proposition 7.5 to $R \subset S$. Since $P \cap A_{1}=(0), P \cap T=(0)$, and so $P_{T}$ survives as a prime in $S$; moreover $\operatorname{rk}(A / P)=\operatorname{rk}\left(S / P_{T}\right)$. Similarly $p_{i} \cap T=(0)$ as $p_{i}$ is a minimal prime; thus $\left(p_{1}\right)_{T}, \ldots,\left(p_{k}\right)_{T}$ are precisely the primes of $R$, and $\operatorname{rk}\left(A_{1} / p_{\mathrm{i}}\right)=\operatorname{rk}\left(\left(R / p_{i}\right)_{T}\right)$. Thus the theorem follows by Proposition 7.5.

We do not know whether it would suffice to assume that $A / P$ is Goldie, rather than $A / P_{G}$ is Goldie.

\section{REFERENCES}

1. S. A. Amitsur, Rings of quotients and Morita contexts, J. Algebra 17 (1971), 273-298.

2. G. Bergman, Groups acting on rings, group graded rings, and beyond (preprint).

3. , On Jacobson radicals of graded rings (preprint).

4. S. Chase, D. Harrison and A. Rosenberg, Galois theory and cohomology of commutative rings, Mem. Amer. Math. Soc., No. 52, 1965.

5. M. Cohen, A Morita context related to finite automorphism groups of rings, Pacific J. Math. 98 (1982), 37-54.

6. M. Cohen and L. Rowen, Group graded rings, Comm. Algebra 11 (1983), 1253-1270.

7. E. C. Dade, Group graded rings and modules, Math. Z. 174 (1980), 241-262.

8. C. Faith, Algebra: rings, modules, and categories. I, Springer-Verlag, New York, 1973.

9. J. M. G. Fell, Induced representations and Banach *-algebraic bundles, Lecture Notes in Math., vol. 582, Springer-Verlag, Berlin and New York, 1977.

10. J. R. Fisher, A Jacobson radical for Hopf module algebras, J. Algebra 34 (1975), 217-231.

11. J. W. Fisher and S. Montgomery, Semiprime skew group rings, J. Algebra 52 (1978), 241-247.

12. I. N. Herstein, Noncommutative rings, Carus Math. Monographs, No. 15, Math. Assoc. Amer., 1968.

13. A. Joseph and L. W. Small, An additivity principle for Goldie rank, Israel J. Math. 31 (1978), $105-114$

14. M. B. Landstad, Duality for dual covariance algebras, Comm. Math. Phys. 52 (1977), 191-202.

15. M. Lorenz and D. S. Passman, Prime ideals in crossed products of finite groups, Israel J. Math. 33 (1979), 89-132.

16. M. Lorenz, S. Montgomery and L. W. Small, Prime ideals in fixed rings. II, Comm. Algebra 10 (1982), 449-455.

17. S. Montgomery, Fixed rings of finite automorphism groups of associative rings, Lecture Notes in Math., vol. 818, Springer-Verlag, Berlin and New York, 1980.

18. __ Prime ideals in fixed rings, Comm. Algebra 9 (1981), 423-449.

19. Y. Nakagami, Dual action on a von Neumann algebra and Takesaki's duality for a locally compact group, Publ. Res. Inst. Math. Sci. 12 (1977), 727-775.

20. Y. Nakagami and M. Takesaki, Duality for crossed products of von Neumann algebras, Lecture Notes in Math., vol. 731, Springer-Verlag, Berlin and New York, 1979.

21. C. Nastacescu, Strongly graded rings of finite groups, Comm. Algebra 11 (1983).

22. W. K. Nicholson and J. F. Waters, Normal radicals and normal classes of rings, J. Algebra 59 (1979), $5-15$.

23. D. S. Passman, The algebraic structure of group rings, Wiley-Interscience, New York, 1977.

24. Fixed rings and integrality, J. Algebra 68 (1981), 510-519.

25. It It's essentially Maschke's theorem, Rocky Mountain J. Math. 13 (1978), 37-54.

26. G. Pedersen, $C^{*}$-algebras and their automorphism groups, LMS Monograph, No. 14, Academic Press, London, 1979. 
27. D. Stratila, D. Voiculescu, and L. Zsido, On crossed products. I, II, Rev. Roumaine Math. Pures Appl. 21 (1976), 1411-1449; ibid 22 (1977), 83-117.

28. M. Sweedler, Hopf algebras, Benjamin, New York, 1969.

29. M. Takesaki, Duality in crossed products and the structure of von Neumann algebras of type III, Acta Math. 131 (1973), 249-310.

Department of Mathematics, Ben Gurion University of the Negev, Beersheva, Israel

Department of Mathematics, University of Southern California, los angeles, California 90089 